PROCEEDINGS OF THE

AMERICAN MA THEMATICAL SOCIE TY

Volume 46, Number 1, October 1974

\title{
A NOTE ON $T_{1}$ TOPOLOGIES
}

\section{WILSON R. CRISLER AND TROY L. HICKS}

ABSTRACT. Let $t$ be a $T_{1}$ topology for a set $X$. The problem of representing $t$ as the lattice product (intersection) of stronger topologies is considered.

It is well known that the set of all topologies on an infinite set $X$ is a complete lattice with partial order $\geq$ defined as stronger than. Also, the family of $T_{1}$ topologies on $X$ is a complete sublattice of $X$. In [1], R. Lalitha proved that: (1) If $t$ is a $T_{1}$ topology for $X$, then $t$ is the lattice product (intersection) of all stronger $T_{2}$ topologies on $X$. (2) If $t$ satisfies the first axiom of countability, it is the intersection of all stronger metric topologies on $X$. In this note, we give a stronger theorem with a much shorter proof. Also, several new results follow from the stronger theorem. Nagata's book [2] is a general reference for notation and terminology.

Theorem 1. If $X$ is an infinite set and $t$ is a $T_{1}$ topology for $X$, then for . every subset $E$ of $X$ such that $E \notin t$ there exists a fully normal, completely normal, disconnected topology $t_{E}$ on $X$ with $t \leq t_{E}$ and $E \notin t_{E}$. Also, if $t$ is first countable, $t_{E}$ is metrizable.

Proof. Given $E \notin t$, choose $p \in E \cap \operatorname{cl}(X-E)$. Let $\Re(p)$ be an open base at $p$. For each $N \in \Re(p)$, set

$$
V_{N}=\Delta \cup[\{p\} \cup(N \cap X-E)] \times[\{p\} \cup(N \cap X-E)]
$$

where $\Delta=\{(x, x): x \in X\}$. Note that $N \cap(X-E) \neq \varnothing$ and

$$
V_{N}[p]=\{p\} \cup(N \cap X-E) \subset N \text {. }
$$

It is easy to see that $\Delta \subset V_{N}, V_{N}=V_{N}^{-1}, V_{N} \cap V_{M}=V_{N \cap M}$, and $V_{N} \circ V_{N}=$ $V_{N^{*}}$. Hence $\mathfrak{B}=\left\{V_{N}: N \in \mathcal{T}_{(p)}\right\}$ is a base for a uniform structure $\mathcal{U}_{\cdot} t_{E}$ is the induced uniform topology.

(1) If $A \subset X$ and $p \notin A$, then $A \in t_{E}$. If $x \in A, x \neq p$ and $t$ is $T_{1}$ implies there exists $N \in \mathcal{N}(p)$ such that $x \notin N$. Then $\{x\}=V_{N}[x] \in t_{E}$.

Presented to the Society, January 28, 1973; received by the editors August 8, 1973 and, in revised form, October 1, 1973.

AMS (MOS) subject classifications (1970). Primary 54D10, 54D15. 
(2) The topology $t_{E}$ is Hausdorff. If $x \neq y$, we may suppose $y \neq p$.

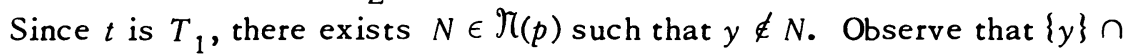
$V_{N}[x]=\varnothing$.

(3) $E \notin t_{E}$. This follows since $p \in E$ and $V_{N}[p]=\{p\} \cup(N \cap X-E) \not \subset E$ for $N \in \Re(p)$.

(4) $t \leq t_{E}$. Suppose $x \in A \in t$. If $x \neq p$, then, by (1), $\{x\} \in t_{E}$. If $x=p$, there exists $N \in \Re(p)$ such that $N \subset A$. Now $V_{N}[p] \subset N \subset A$. Thus $A \in t_{E}$.

(5) $t_{E}$ is completely normal. Suppose $A \cap \bar{B}=\bar{A} \cap B=\varnothing$. If $p \notin A \cup$ $B$, then, by (1), $A \in t_{E}$ and $B \in t_{E}$. Clearly, $A \cap B=\varnothing$. If $p \in A \cup B$, suppose $p \in A$. Then $p \notin B$ implies $B \in t_{E} \cdot X-\bar{B} \in t_{E}, A \subset X-\bar{B}$, and $B \cap$ $X-\bar{B}=\varnothing$.

(6) $t_{E}$ is fully normal. Let $\mathcal{U}=\left\{O_{\alpha}: \alpha \in I\right\}$ be an open cover of $X$. $p \in O_{a^{\prime}}$ for some $\alpha^{\prime} \in I . X=O_{a^{\prime}} \cup\left(\bigcup_{x \neq p}\{x\}\right)$. Denote this open cover by B. $\mathfrak{B}^{\Delta}<\mathcal{U}$. For, if $x \in X$ and $x \neq p$, then $S(x, \mathfrak{B})=O_{\alpha^{\prime}}$ if $x \in O_{a^{\prime}}$ and $S(x, \Re)=\{x\}$ if $x \notin O_{a^{\prime}}$. Also, $S(p, \Re)=O_{a^{\prime}}$.

(7) $t_{E}$ is disconnected. Since $t$ is $T_{1}$, there exists $N \in \Re(p)$ with $N \neq X . V_{N}[p] \subset N$ implies there exists $O \in t_{E}$ with $p \in O \neq X . p \in O$ implies $\bar{O}=0$.

(8) $t$ is first countable implies $t_{E}$ is metrizable. In this case, we choose $\Re(p)$ so it is countable and clearly the uniform structure $\mathcal{U}$ has a countable base. Thus $t_{E}$ is metrizable.

Corollary 1. If $t$ is a $T_{1}$ topology on an infinite set, then $t=\bigcap\{u: u$ is $T_{2}$ and $\left.t \leq u\right\}=\bigcap\{u: u$ is regular and $t \leq u\}=\bigcap\{u: u$ is normal and $t \leq u\}=$ $\bigcap\{u: u$ is fully normal, completely normal, disconnected, and $t \leq u\}$.

Corollary 2. If $t$ is the weakest $T_{1}$ topology on an infinite set, then $t=\bigcap\left\{u: u\right.$ is $\left.T_{1}\right\}=\bigcap\left\{u: u\right.$ is $\left.T_{2}\right\}=\bigcap\{u: u$ is regular $\}=\bigcap\{u: u$ is fully normal, completely normal, and disconnected $\}$.

The reader can observe other consequences of the theorem. For example, if $t$ is a $T_{2}$ topology on an infinite set, then $t=\bigcap\{u: u$ is regular and $t \leq u\}$. Actually, the topology $t_{E}$ in the theorem has other properties. We give one in the following

Lemma. The uniform structure $\mathcal{U}$ that gives $t_{E}$ is complete.

Proof. Suppose $\mathcal{F}$ is a $\mathcal{U}$-Cauchy filter and let $N \in \mathcal{N}(p)$. Then there exists $F \in \mathcal{F}$ such that $F \times F \subset V_{N} \cdot F \neq \varnothing$. If $F=\left\{x_{0}\right\}, \mathcal{F}$ converges to $x_{0}$. If $F \neq\left\{x_{0}\right\}, F \supset\{a, b\}$ where $a \neq b$, and we show that $F \subset N$. Then 
$N \in \mathcal{F}$ and $\mathcal{F}$ converges to $p$. If $c \in F$ and $c \neq p$, choose $d \in F$ such that $c \neq d$. Then $(c, d) \in F \times F \subset V_{N}$ implies $c \in N \cap X-E \subset N$.

The construction, given in the proof of Theorem 1, involves only elementary concepts. Also, it is useful in constructing examples. We illustrate this fact.

Example. $X$ is the unit interval $[0,1], t$ is the topology of finite complements, and $E=\{O\} \notin t . p=O \in E \cap \operatorname{cl}(X-E)$. If $O \in N \in t$

$$
V_{N}[O]=\{O\} \cup(N \cap X-\{O\})=N \text {. }
$$

Thus the $t_{E}$ open sets containing $O$ are the $t$ open sets containing $O$ and

$$
t_{E}=\{\varnothing, X\} \cup\{A: O \dot{\notin} A\} \cup\{B: O \in B \text { and } X-B \text { is finite }\} .
$$

(1) From the proof of Theorem $1, t_{E}$ is uniformizable, completely normal, fully normal, and disconnected. It is clearly compact.

(2) We show that $t_{E}$ is not pseudo-metrizable and also not perfectly normal. If it were pseudo-metrizable it would be metrizable since $t_{E}$ is $T_{2}$. Also, we could write $\{O\}=\bigcap_{n=1}^{\infty} A_{n}$ where $O \in A_{n} \in t_{E}$. Then $X-\{O\}=$ $\bigcup_{n=1}^{\infty}\left(X-A_{n}\right)$ would be countable which is impossible. Since $X-\{O\} \epsilon$ $t_{E},\{O\}$ is closed and the above argument shows that $t_{E}$ is not perfectly normal.

\section{REFERENCES}

1. R. Lalitha, $A$ note on the lattice structure of $T_{1}$ topologies on an infinite set, Math. Student 35 (1967), 29-33. MR 40 \#6484.

2. Jun-iti Nagata, Modern general topology, Bibliotheca Mathematica, vol. 7, North-Holland, Amsterdam; Wolters-Noordhoff, Groningen; Interscience, New York, 1968. MR 41 \#9171.

DEPARTMENT OF MATHEMATICS, UNIVERSITY OF MISSOURI, ROLLA, MISSOURI 65401 